\title{
Experimental realization of diffusion with
}

\section{stochastic resetting}

\author{
Ofir Tal-Friedman, ${ }^{\dagger}$ Arnab Pal, ${ }^{*} \ddagger$ Amandeep Sekhon, ${ }^{\ddagger}$ Shlomi Reuveni, ${ }^{*}+$ and \\ Yael Roichman*, $\dagger, \ddagger$ \\ $\dagger$ School of Physics \& Astronomy, Raymond and Beverly Sackler Faculty of Exact Sciences, Tel \\ Aviv University, Tel Aviv 6997801, Israel \\ $\ddagger$ School of Chemistry, The Center for Physics and Chemistry of Living Systems, \& The Mark \\ Ratner Institute for Single Molecule Chemistry, Tel Aviv University, Tel Aviv 6997801, Israel \\ E-mail: arnabpal@mail.tau.ac.il; shlomire@tauex.tau.ac.il; roichman@tauex.tau.ac.il
}

\begin{abstract}
Stochastic resetting is prevalent in natural and man-made systems giving rise to a long series of non-equilibrium phenomena. Diffusion with stochastic resetting serves as a paradigmatic model to study these phenomena, but the lack of a well-controlled platform by which this process can be studied experimentally has been a major impediment to research in the field. Here, we report the experimental realization of colloidal particle diffusion and resetting via holographic optical tweezers. We provide the first experimental corroboration of central theoretical results, and go on to measure the energetic cost of resetting in steady-state and first-passage scenarios. In both cases, we show that this cost cannot be made arbitrarily small due to fundamental constraints on realistic resetting protocols. The methods developed herein open the door to future experimental study of resetting phenomena beyond diffusion.
\end{abstract}




\section{Introduction}

Stochastic resetting is ubiquitous in nature, and has recently been the subject of vigorous stud-

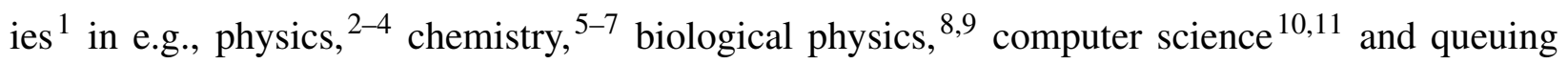
theory. ${ }^{[2] 13}$ A stylized model to study resetting phenomena was proposed by Evans and Majumdar. ${ }^{[2}$ The model, which considers a diffusing particle subject to stochastic resetting, exhibits many rich properties e.g., the emergence of a non-equilibrium steady state and interesting relaxation dynamics $^{2-4-14-18}$ which were also observed in other systems with stochastic resetting. ${ }^{19-27}$ The model is also pertinent to the study of search and first-passage time (FPT) questions. ${ }^{[28129}$ In particular, it was used to show that resetting can significantly reduce the mean FTP of a diffusing particle to a target by mitigating the deleterious effect of large FPT fluctuations that are intrinsic to diffusion in the absence of resetting. $1-4 \mid-17730-32]$ Interestingly, this beneficial effect of resetting also extends beyond diffusion and applies to many other stochastic processes; $\frac{1120+2212612733-40}{4-4}$ and further studies moreover revealed a genre of universality relations associated with optimally restarted processes as well as the existence of a globally optimal resetting strategy.

Despite a long catalogue of theoretical studies on stochastic resetting, no attempt to experimentally study resetting in a controlled environment has been made to date, (but see very recent work that appeared after our arXiv submission ${ }^{41}$ ). This is needed as resetting in the real world is never 'clean' as in theoretical models which glance over physical complications for the sake of analytical tractability and elegance. In this letter, we report the experimental realization of diffusion with stochastic resetting (Fig. 11). Our setup comprises of a colloidal particle suspended in fluid (in quasi-two dimensions) and resetting is implemented via a home built holographic optical tweezers (HOTs) system ${ }^{42+45}$ described in the Supporting Information accompanying this letter. ${ }^{[6}$ We study two, physically amenable, resetting protocols in which the particle is returned to the origin: (i) at a constant velocity, and (ii) within a constant time. In both cases, resetting is stochastic: time intervals between resetting events come from an exponential distribution with mean $1 / r$.

Every experiment starts by drawing a series of random resetting times $\left\{t_{1}, t_{2}, t_{3}, \ldots\right\}$ taken from an exponential distribution with mean $1 / r$. At time zero, the particle is trapped at the origin and 
a)

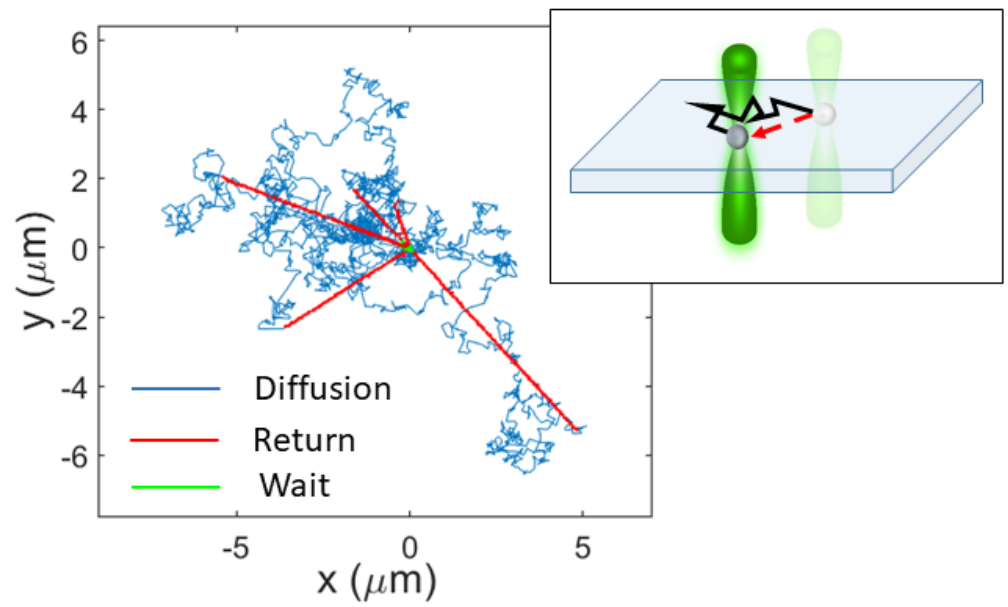

b)

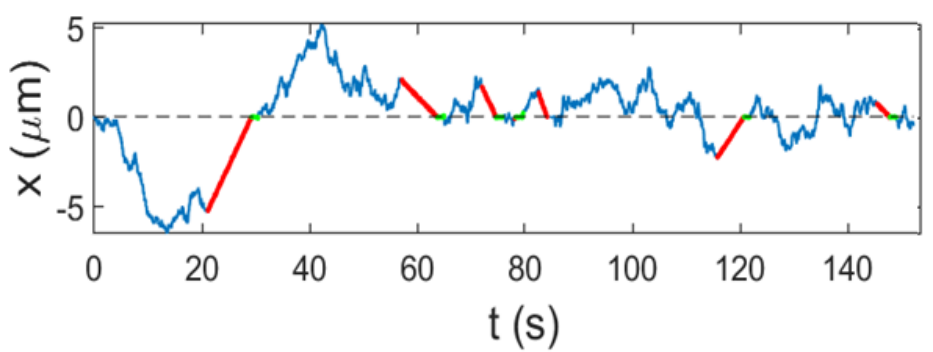

Figure 1: Experimental realization of diffusion with stochastic resetting. a) A sample trajectory of a silica particle diffusing (blue) near the bottom of a sample cell. The particle sets off from the origin and is reset at a rate $r=0.05 \mathrm{~s}^{-1}$. Following a resetting epoch, the particle is driven back to the origin at a constant radial velocity $v=0.8 \mu \mathrm{m} / \mathrm{s}$ using HOTs (red). After the particle arrives at the origin it remains trapped there for a short period of time to improve localization (green). Inset shows a schematic illustration of the experiment. b) Projection of the particle's trajectory onto the $x$-axis.

the experiment, which consists of a series of statistically identical steps, begins. At the $i$-th step of the experimental protocol, the particle is allowed to diffuse for a time $t_{i}$ eventually arriving at a position $\left(x_{i}, y_{i}\right)$. At this time, an optical trap is projected onto the particle and the particle is dragged by the trap to its initial position. A typical trajectory of a colloidal particle performing diffusion under stochastic resetting with $r=0.05 s^{-1}$ is shown in Fig. 17a and Supplementary movie $1 .{ }^{46}$ Note that the trajectory is composed of three phases of motion: diffusion, return, and a short waiting time to allow for optimal localization at the origin (Fig.1p).

Below we utilize our setup to study the long time position distribution of a tagged particle and its dependence on the resetting protocol. We consider the energetic cost of resetting and character- 
ize the mean and distribution of energy spent per resetting event. Finally, we study the mean FPT of a tagged particle to a region in space and the energetic cost of resetting in this scenario. We conclude with discussion and outlook on the future of experimental studies of stochastic resetting.

\section{Stochastic resetting with instantaneous returns}

We first study the case in which upon resetting the particle is teleported back to the origin in zero time. This case was the first to be analysed theoretically, ${ }^{2}$ thus providing a benchmark for experimental results. A particle undergoing free Brownian motion is not bound in space. It has a Gaussian position distribution with a variance that grows linearly with time. Repeated resetting of the particle to its initial position will, however, result in effective confinement and in a nonGaussian steady state distribution: $\rho(x)=\frac{\alpha_{0}}{2} e^{-\alpha_{0}|x|}$, where $\alpha_{0}=\sqrt{r / D}$, and $D$ is the diffusion constant. ${ }^{233}$ Estimating the steady state distribution of the particle's position along the $x$-axis by digitally removing the return (red) and wait (green) phases of motion in Fig. 1, 146 we find that the experimentally measured results conform well with this theoretical prediction (Fig. 2 a). The steady state radial density of the particle can also be extracted from the experimental trajectories by looking at the steady-state distribution of the distance $R=\sqrt{x^{2}+y^{2}}$ from the origin. Here too, we find excellent agreement with theory (Fig. $2 \mathrm{p}$ ).

\section{Stochastic resetting with non-instantaneous returns}

We now turn our attention to more realistic pictures of diffusion with stochastic resetting. These have just recently been considered theoretically in attempt to account for the non-instantaneous

returns and waiting times that are seen in all physical systems that include resetting. $.5-7 / 20|24-27 / 40| 47$ First, we consider a case where upon resetting HOTs are used to return the particle to the origin at a constant radial velocity $v=\sqrt{v_{x}^{2}+v_{y}^{2}}$ (Fig. 11. This case naturally arises for resetting by constant 

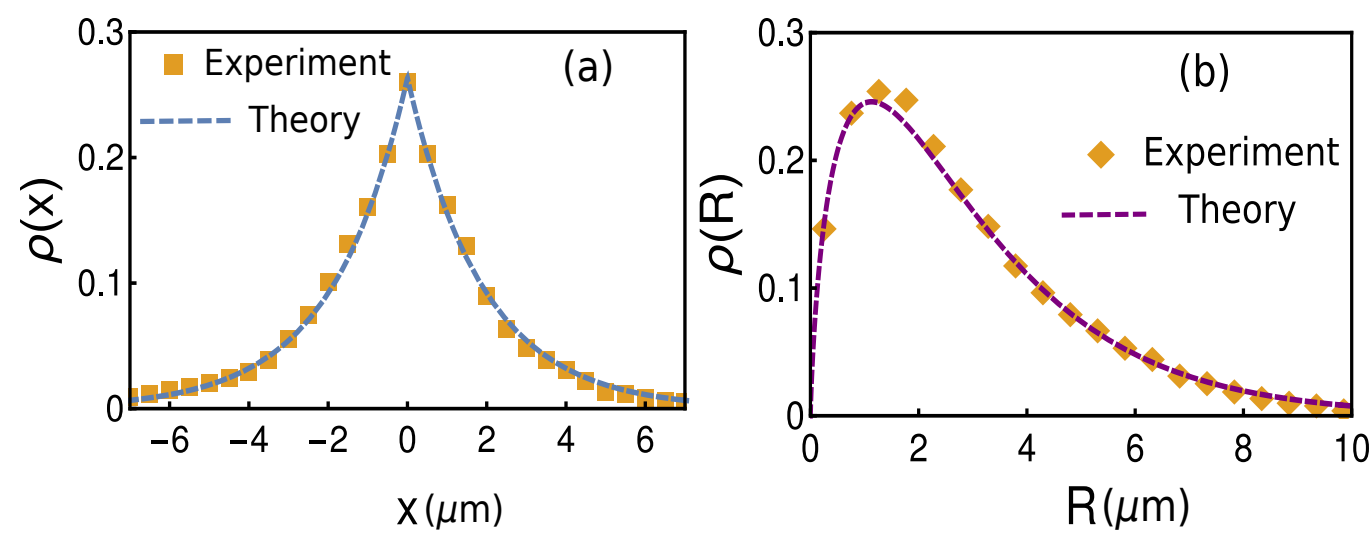

Figure 2: Steady-state distribution of diffusion with stochastic resetting and instantaneous returns. a) Distribution of the position along the $x$-axis. Markers come from experiments and the dashed line is the theoretical prediction $\rho(x)=\frac{\alpha_{0}}{2} e^{-\alpha_{0}|x|}$, where $\alpha_{0}=\sqrt{r / D}$, and $D$ is the diffusion constant. b) The radial position distribution. Markers come from experiments and the dashed line is the theoretical prediction $\rho(R)=\alpha_{0}^{2} R K_{0}\left(\alpha_{0} R\right)^{\underline{46}}$ with $K_{n}(z)$ standing for the modified Bessel function of the second kind. ${ }^{48}$ In both panels no fitting procedure was applied: $D=0.18 \pm 0.02 \mu \mathrm{m}^{2} / \mathrm{s}$ was measured independently and $r=0.05 s^{-1}$ was set by the operator.

force in the over-damped limit. We find that the radial steady state density is then given by $\underline{46}$

$$
\rho(R)=p_{D}^{c . v \cdot} \rho_{\mathrm{diff}}(R)+\left(1-p_{D}^{c . v \cdot}\right) \rho_{\mathrm{ret}}(R)
$$

where $p_{D}^{c . v .}=\left(1+\frac{\pi r}{2 \alpha_{0} v}\right)^{-1}$ is the steady-state probability to find the particle in the diffusive phase. Here, $\rho_{\text {diff }}(R)=\alpha_{0}^{2} R K_{0}\left(\alpha_{0} R\right)$ and $\rho_{\text {ret }}(R)=\frac{2 \alpha_{0}^{2}}{\pi} R K_{1}\left(\alpha_{0} R\right)$ stand for the conditional probability densities of the particle's position when in the diffusive and return phases respectively, and $K_{n}(z)$ is the modified Bessel function of the second kind. ${ }^{48}$ Bessel functions naturally appear here due to the rotational symmetry of the process and the resetting protocol. The result in Eq. (1) is in very good agreement with experimental data as shown in Fig. 3 a and Fig. S3. We note that the theoretical result (1) was also derived in $\frac{49}{4}$ using an alternative method.

Next, we consider a case where HOTs are used to return the particle to the origin at a constant time $\tau_{0}$. This case is appealing due to its simplicity. Here, we find that the radial steady-state position distribution reads 46

$$
\rho(R)=p_{D}^{\text {c.t. }} \rho_{\mathrm{diff}}(R)+\left(1-p_{D}^{\text {c.t. }}\right) \rho_{\mathrm{ret}}(R)
$$



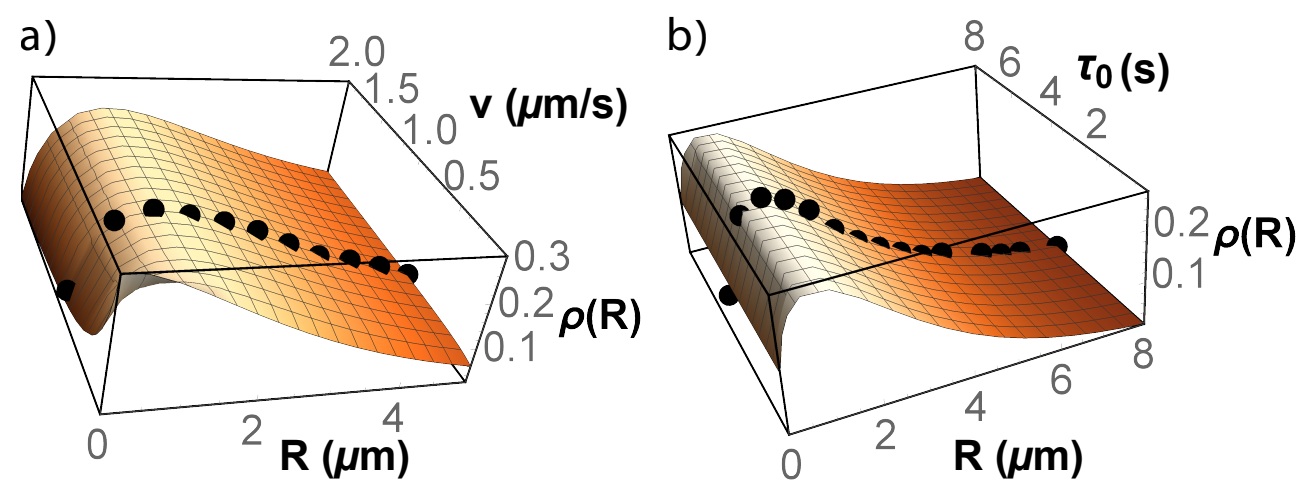

Figure 3: Steady-state distributions of diffusion with stochastic resetting and non-instantaneous returns. a) The radial position distribution, $\rho(R)$, vs. the distance $R$ and the radial return velocity $v$ as given by Eq. (1). Experimental results obtained for $v=0.8 \mu \mathrm{m} / \mathrm{s}$ are superimposed on the theoretical prediction (black spheres). b) The radial position distribution vs. $R$ and the return time $\tau_{0}$ as given by Eq. (2). Experimental results obtained for $\tau_{0}=3.79 \mathrm{~s}$ are superimposed on the theoretical prediction (black spheres).

where $p_{D}^{\text {c.t. }}=\left(1+r \tau_{0}\right)^{-1}$ is the steady-state probability to find the particle in the diffusive phase, and with $\rho_{\text {diff }}(R)=\alpha_{0}^{2} R K_{0}\left(\alpha_{0} R\right)$ and $\rho_{\text {ret }}(R)=\frac{\pi \alpha_{0}^{2}}{2}\left[\frac{1}{\alpha_{0}}-R\left[K_{0}\left(\alpha_{0} R\right) L_{-1}\left(\alpha_{0} R\right)+K_{1}\left(\alpha_{0} R\right) L_{0}\left(\alpha_{0} R\right)\right]\right]$, standing for the conditional probability densities of the particle's radial position when in the diffusive and return phases respectively. Here, $\boldsymbol{L}_{n}$ is the modified Struve function of order $n \cdot{ }^{48}$ The result in Eq. (2) is in very good agreement with experimental data as shown in Fig. 3b and Fig. S5. Note that Eq. (1) and Eq. (2) interpolate between the limit of instantaneous returns, with $v \rightarrow \infty$ or $\tau_{0} \rightarrow 0$, and the case of infinitely slow returns where $\rho(R)$ is dominated by the return statistics. Indeed, we find that short return times and high return velocities are similar as returns are effectively instantaneous, while in the other extreme marked differences are observed (Fig. S4 and S6).

\section{Energy cost per resetting}

A central, and previously unexplored, aspect of stochastic resetting is the energetic cost associated with the resetting process itself. As discussed above, stochastic resetting prevents a diffusing particle from spreading over the entire available space as it normally would. Instead, a localized, non-equilibrium, steady-state is formed; but the latter can only be maintained by working on the system continuously. 
a)

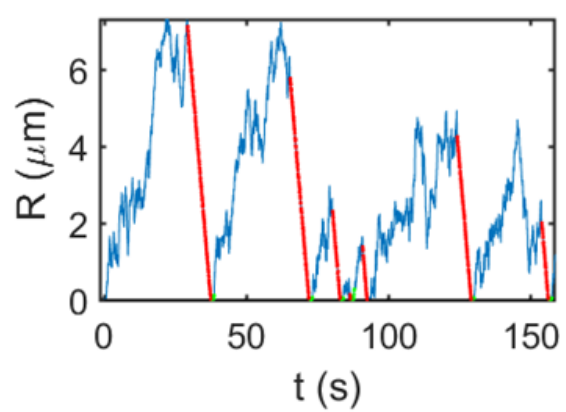

b)

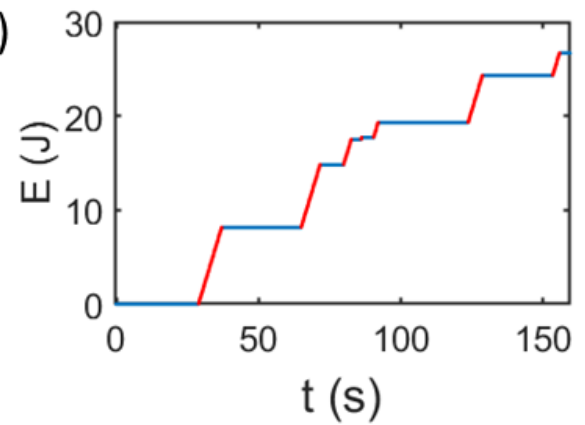

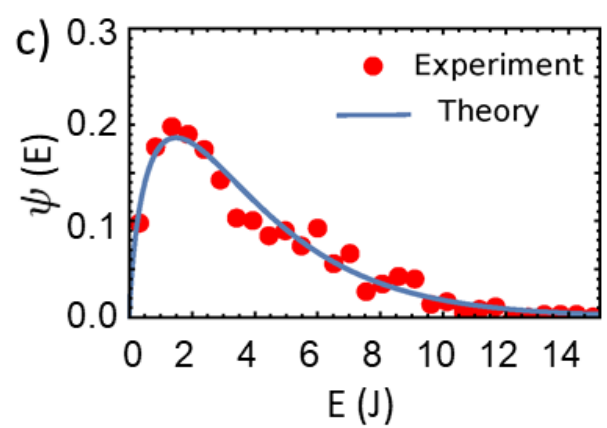

d)

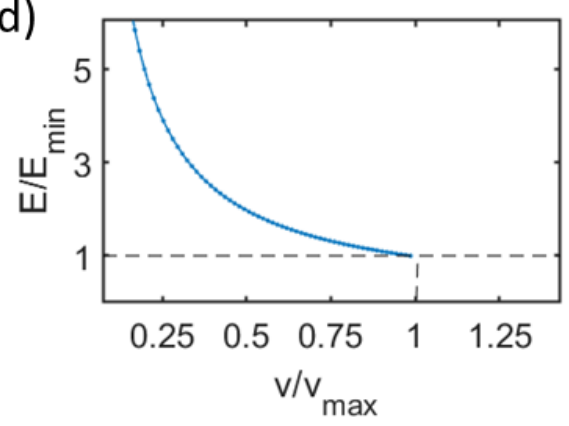

Figure 4: Energetic cost of resetting. a) The radial distance from the origin vs. time for a particle diffusing with a resetting rate $r=0.05 \mathrm{~s}^{-1}$ and constant radial return velocity $v=0.8 \mu \mathrm{m} / \mathrm{s}$. b) The cumulative energy expenditure for the trajectory in panel a) (neglecting the cost of the wait period). c) The distribution of energy spent per resetting event. Red disks come from experiments and the theoretical prediction of Eq. (4) is plotted as a solid blue line. d) Normalized energy spent per resetting event at constant power vs. the normalized radial return velocity as given by Eq. (5). The minimal energy is attained at a maximal velocity for which the trap is just barely strong enough to prevent the particle from escaping.

In our experiments, work is done by the laser to capture the particle in an optical trap and drag it back to the origin. The total energy spent per resetting event is then simply given by $E=\mathscr{P} \tau(R)$, where $\mathscr{P}$ is the laser power fixed at $1 \mathrm{~W}$ and $\tau(R)$ is the time required for the laser to trap the particle at a distance $R$ and bring it back to the origin. As the particle's distance at the resetting epoch fluctuates randomly from one resetting event to another (Fig. $4 \mathrm{a}$ ), the energy spent per resetting event is also random (Fig. 4p). To compute its distribution, we note that $E$ is proportional to the return time whose probability density function is in turn given by $\underline{46}$

$$
\phi(t)=\int_{\mathscr{D}} d \vec{R} \delta[t-\tau(\vec{R})] \int_{0}^{\infty} d t^{\prime} f\left(t^{\prime}\right) G_{0}\left(\vec{R}, t^{\prime}\right)
$$

Here, $f(t)$ is the probability density governing the resetting time, $\vec{R}$ is the $d$-dimensional position 
vector, $\tau(\vec{R})$ is the return time and $G_{0}(\vec{R}, t)$ is the propagator of the underlying stochastic dynamics. In our experimental setup, we have $f(t)=r e^{-r t}$ and $G_{0}(R, t)=\frac{1}{4 \pi D t} e^{-R^{2} / 4 D t}$ which is the diffusion propagator in polar coordinates. Moreover, in the case of constant radial return velocity $v$, we have $\tau(\vec{R})=R / v$. A derivation then yields the probability density of the energy spent per resetting event $^{46}$

$$
\psi(E)=\frac{E}{E_{0}^{2}} K_{0}\left(E / E_{0}\right)
$$

with $E_{0}=\alpha_{0}^{-1} v^{-1} \mathscr{P}$; and note that this is a special case of the K-distribution. $\underline{50[51}$ The mean energy spent per resetting event can be computed directly from Eq. (4) and is given by $\langle E\rangle=$ $\pi E_{0} / 2$. Eq. (4) demonstrates good agreement with experimental data (Fig. 4c).

As $\langle E\rangle \propto v^{-1}$, it can be made smaller by working at higher return velocities. However, the stiffness, $k$, of the optical trap must be strong enough to oppose the drag force acting on the particle so as to keep it in the trap. Assuming the maximum allowed displacement of a particle in the trap is $\approx 0.5 \mu \mathrm{m},{ }^{52}$ we find that working conditions must obey $k \geq 2 \gamma v$. As the stiffness is proportional to the laser power, $k=\mathscr{C} \mathscr{P}$ (where $\mathscr{C}$ is the conversion factor), the maximal working velocity is given by $v_{\max } \approx \frac{1}{2} \mathscr{C} \mathscr{P} / \gamma$ which-independent of laser power-minimizes energy expenditure to $E_{\min } \approx \pi \gamma \mathscr{C}^{-1} \alpha_{0}^{-1}$. Going to dimensionless variables we find

$$
\langle E\rangle / E_{\min }=v_{\max } / v
$$

for $v<v_{\max }$. This nicely illustrates that $\langle E\rangle$ cannot be lowered indefinitely, i.e., that there is a minimal energy cost per resetting event (Fig. 4d).

\section{Energy cost per first-passage}

Having looked at stationary properties of diffusion with resetting, we now turn attention to firstpassage properties which have numerous applications. $4-9 \mid 28[29[33-40[53-65]$ We recall that while the 
mean first-passage time (MFPT) of a Brownian particle to a stationary target diverges, $\stackrel{28 \mid 29}{2}$ resetting will render it finite, ${ }^{2}$ even if returns are non-instantaneous. $\frac{5+7 / 20[26|40|}{}$ To experimentally show this, we consider the setup in Fig. 5 a.

A first passage experiment starts at time zero when the particle is at the origin. Resetting is conducted stochastically with rate $r$, and HOTs are used to return the particle to the origin at a constant return time $\tau_{0}$. However, we now also define a target, set to be a virtual infinite absorbing wall located at $x=L$, i.e., parallel to the $y$-axis. The particle is allowed to diffuse with stochastic resetting until it hits the target, and the hitting times (FPTs) are recorded (Fig. 5b). A typical trajectory extracted from such an experiment with $\tau_{0}=3.79 \mathrm{~s}, L=1 \mu \mathrm{m}$, and $r=0.05 \mathrm{~s}^{-1}$ is shown in Fig. 5b, Fig. S7, and Supplementary movie 2. Measurements were also taken for: $r=0.0667 s^{-1}$, $0.125 s^{-1}, 0.5 s^{-1}$, and $1 s^{-1} .46$

To check agreement between experimental FPT data and theory, we derived a formula for the mean FPT of diffusion with stochastic resetting and constant time returns 46

$$
\left\langle T_{r}\right\rangle=\left(\frac{1}{r}+\tau_{0}\right)\left[e^{\sqrt{r L^{2} / D}}-1\right] .
$$

Eq. (6) is in excellent agreement with data as shown in Fig. 5c, including accurate prediction of the optimal resetting rate which minimizes the mean FPT of the particle to the target.

As resetting requires energy, lowering the mean FPT will have a cost—which to date has been completely ignored. To compute it, we require the probability density of the return time in a FPT scenario which is generally given by 46

$$
\phi_{\mathrm{FP}}(t)=\frac{1}{p} \int_{\mathscr{D}} d \vec{R} \delta[t-\tau(\vec{R})] \int_{0}^{\infty} d t^{\prime} f\left(t^{\prime}\right) G_{\mathrm{abs}}\left(\vec{R}, t^{\prime}\right)
$$

where $p$ is the probability that a reset event will occur before a first passage event and $G_{\text {abs }}(\vec{R}, t)$ is the reset-free propagator in the presence of the absorbing target. As the number of resets per first passage event is geometrically distributed with mean $p /(1-p)$, one can compute, $\left\langle E_{\mathrm{FP}}\right\rangle$, the average energy spent per first passage event. ${ }^{46}$ Setting $\tau(\vec{R})=\tau_{0}$, we find $\left\langle E_{\mathrm{FP}}\right\rangle=\mathscr{P} \tau_{0}\left(e^{\sqrt{r L^{2} / D}}-1\right)$ 
a) virtual absorbing wall
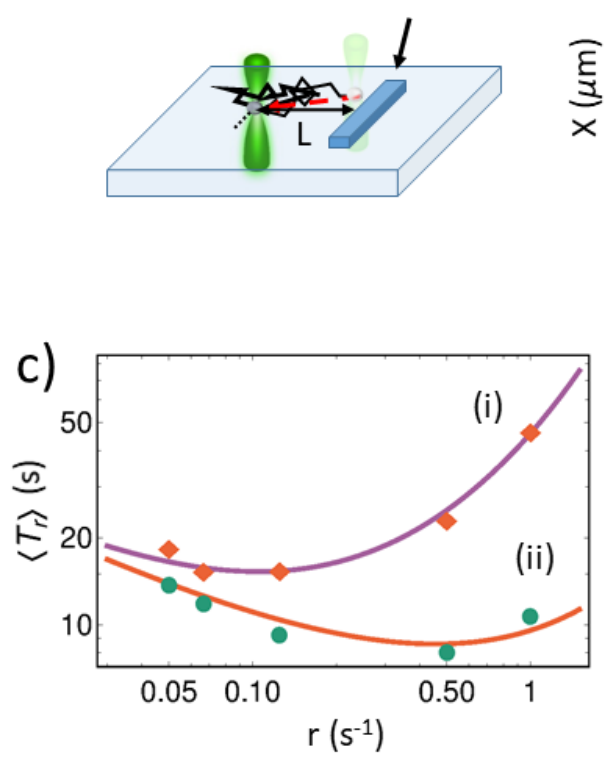
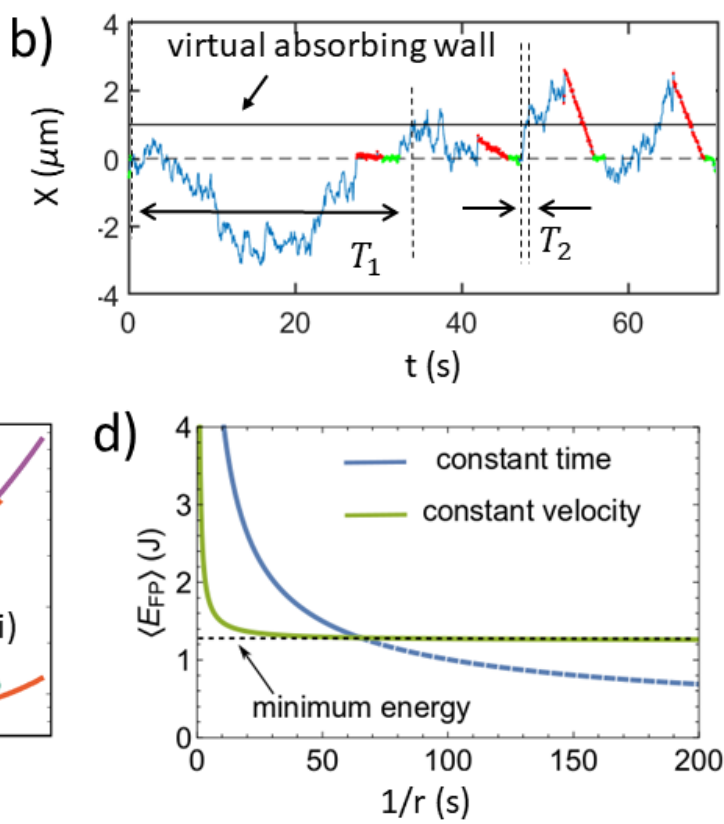

Figure 5: a) Schematic illustration of a first-passage experiment. The target is a virtual absorbing wall. b) Projection of the particle's trajectory onto the $x$-axis. The position of the wall is marked as a solid line. Returns are marked in red (return time $\tau_{0}=3.79 \mathrm{~s}$ ) and waiting periods are marked in green. The first two first-passage times are marked by $T_{1}$ and $T_{2}$. c) The mean FPT to the wall vs. the resetting rate for: (i) non-instantaneous returns with $\tau_{0}=3.79 \mathrm{~s}$; and (ii) instantaneous returns $\left(\tau_{0}=0\right)$. Theoretical predictions [Eq. (6), solid lines] are in good agreement with experimental data (symbols). d) Energy cost per first-passage event for the constant time (blue, $\tau_{0}=3.79 \mathrm{~s}$ ) and constant velocity (green, $v=0.8 \mu \mathrm{m} / \mathrm{s}$ ) return protocols. For a fixed laser power, the energetic cost of the constant velocity protocol is bounded from below. In the constant time protocol, we have dashed the range of resetting rates where the average return velocity is higher than $v .{ }^{46}$ The latter cannot exceed $v_{\max }$, thus bounding the energy cost of the constant time protocol.

which vanishes as $r \rightarrow 0$ (Fig. 5d). ${ }^{[46}$ Note, however, that in this limit $|\vec{R}|$ can be very large at the resetting moment which inevitably implies frequent cases where $|\vec{R}| / \tau_{0}>v_{\max }$. This in turn results in particles escaping the optical trap and in utter breakdown of the constant return time protocol. 46 To avoid this problem, we instead consider the more realistic constant velocity protocol which gives $\left\langle E_{\mathrm{FP}}\right\rangle=\frac{\mathscr{P} L}{v}\left[\frac{2 \sinh \alpha_{0} L}{\alpha_{0} L}-1\right]$, for $v<v_{\max }$ (Fig. $5 \mathrm{~d}$ ). This result surprisingly reveals a dynamical transition: while $\left\langle E_{\mathrm{FP}}\right\rangle \equiv 0$ when $r=0$, for all $r>0$ one has $\left\langle E_{\mathrm{FP}}\right\rangle>\mathscr{P} L / v$, which means that the energy spent per FPT event cannot drop below that which is required to drag the particle directly to $L$ at a constant velocity $v$. Setting $v=v_{\max }$ in the above bound gives $\left\langle E_{\mathrm{FP}}\right\rangle>2 L \gamma / \mathscr{C}$, which does not depend on laser power or return velocity. 


\section{Discussion and future outlook}

In this study, we have demonstrated a unique and versatile method to realize experimentally a resetting process in which many parameters can be easily controlled. To test our platform, we first used it to experimentally corroborate existing theoretical predictions, which in turn motivated experimental and theoretical study of novel and more realistic aspects of diffusion with stochastic resetting. Of prime importance in this regard is the energetic cost of resetting, ${ }^{66}$ which we have characterized in both the steady-state and first-passage settings. Combining analytically derived expressions with the physics of resetting via HOTs then surprisingly revealed lower bounds on the energy spent per resetting for steady state and first passage events. Our results were based on Eqs. (3) and (7) which are general and can be used as platform to extend our findings to a wide range of stochastic motions, resetting time distributions, return protocols, and arbitrary dimensions. In addition, our setup can be easily adapted to experimentally explore regimes that are well beyond the reach of existing theories of stochastic resetting, e.g., multibody systems with strong interactions. These will be considered elsewhere.

Finally, we note that the optical trapping method used herein is far from being the most efficient way to apply force to a colloidal particle. In fact, in our experiments we used $1 W$ of power at the laser output to create a trap of $k=30 \mathrm{pN} / \mu \mathrm{m}$ for a silica bead of radius $a=0.75 \mu \mathrm{m}$. For experiments with a constant return velocity $v=0.8 \mu \mathrm{m} / \mathrm{s}$ and resetting rate $r=0.05 \mathrm{~s}^{-1}$, the average return time was $\langle\tau(R)\rangle=\pi \alpha_{0}^{-1} v^{-1} / 2=3.68 s$, where the average was done with respect to $\phi(t)$ using Eq. (3). This translates to an average energy expenditure of $\langle E\rangle=\mathscr{P}\langle\tau(R)\rangle=3.68 \pm 0.05 J$ per resetting event. In contrast, the work done against friction to drag the particle at a constant velocity $v$ for a distance $R$ is given by $W_{\text {drag }}=\gamma \nu R$ where $\gamma=6 \pi \eta a$ is the the Stokes drag coefficient. Taking averages, we find $\langle R\rangle=v\langle\tau(R)\rangle=\pi \alpha_{0}^{-1} / 2$. The work required per resetting event is then given by $\left\langle W_{\text {drag }}\right\rangle=\gamma v\langle R\rangle=\pi \alpha_{0}^{-1} \gamma v / 2$, which translates into $3.4 \cdot 10^{-20} J$ or $8.3 k_{B} T$ per resetting event. We thus see that $\left\langle W_{\text {drag }}\right\rangle \ll\langle E\rangle$, i.e., that the work required to reset the particle's position is orders of magnitude smaller than the actual amount of energy spent when resetting is done using HOTs. Developing energy efficient resetting methods is a future challenge. 


\section{Acknowledgement}

The authors acknowledge Gilad Pollack for his help in coding the resetting protocol of the HOTs. A. P. acknowledges support from the Raymond and Beverly Sackler Post-Doctoral Scholarship at Tel-Aviv University; and Somrita Ray for many fruitful discussions. A. S. acknowledges support from the Ratner center for single molecule studies. S. R. acknowledges support from the Azrieli Foundation, from the Raymond and Beverly Sackler Center for Computational Molecular and Materials Science at Tel Aviv University, and from the Israel Science Foundation (grant No. 394/19). Y. R. acknowledges support from the Israel Science Foundation (grant No. 988/17).

\section{Supporting Information Available}

The following files are available free of charge.

See Supplementary Information for the details of the theoretical derivations, experimental methods, and other results.

\section{Author contributions}

Ofir Tal Friedman, Arnab Pal and Amandeep Sekhon have equally contributed to this work.

\section{References}

(1) Evans, M.R., Majumdar, S.N. and Schehr, G., 2020. Stochastic resetting and applications. Journal of Physics A: Mathematical and Theoretical.

(2) Evans, M.R. and Majumdar, S.N., 2011. Diffusion with stochastic resetting. Physical review letters, 106(16), p.160601.

(3) Evans, M.R. and Majumdar, S.N., 2011. Diffusion with optimal resetting. Journal of Physics A: Mathematical and Theoretical, 44(43), p.435001. 
(4) Evans, M.R., Majumdar, S.N. and Mallick, K., 2013. Optimal diffusive search: nonequilibrium resetting versus equilibrium dynamics. Journal of Physics A: Mathematical and Theoretical, 46(18), p.185001.

(5) Reuveni, S., Urbakh, M. and Klafter, J., 2014. Role of substrate unbinding in MichaelisMenten enzymatic reactions. Proceedings of the National Academy of Sciences, 111(12), pp.4391-4396.

(6) Rotbart, T., Reuveni, S. and Urbakh, M., 2015. Michaelis-Menten reaction scheme as a unified approach towards the optimal restart problem. Physical Review E, 92(6), p.060101.

(7) Robin, T., Reuveni, S. and Urbakh, M., 2018. Single-molecule theory of enzymatic inhibition. Nature communications, 9(1), p.779.

(8) Roldan, E., Lisica, A., Sanchez-Taltavull, D. and Grill, S.W., 2016. Stochastic resetting in backtrack recovery by RNA polymerases. Physical Review E, 93(6), p.062411.

(9) Budnar, S., Husain, K.B., Gomez, G.A., Naghibosadat, M., Varma, A., Verma, S., Hamilton, N.A., Morris, R.G. and Yap, A.S., 2019. Anillin promotes cell contractility by cyclic resetting of RhoA residence kinetics. Developmental cell, 49(6), pp.894-906.

(10) Luby, M., Sinclair, A. and Zuckerman, D., 1993. Optimal speedup of Las Vegas algorithms. Information Processing Letters, 47(4), pp.173-180.

(11) Gomes, C.P., Selman, B. and Kautz, H., 1998. Boosting combinatorial search through randomization. AAAI/IAAI, 98, pp.431-437.

(12) Di Crescenzo, A., Giorno, V., Nobile, A.G. and Ricciardi, L.M., 2003. On the M/M/1 queue with catastrophes and its continuous approximation. Queueing Systems, 43(4), pp.329-347.

(13) Kumar, B.K. and Arivudainambi, D., 2000. Transient solution of an M/M/1 queue with catastrophes. Computers \& Mathematics with applications, 40(10-11), pp.1233-1240. 
(14) Evans, M.R. and Majumdar, S.N., 2014. Diffusion with resetting in arbitrary spatial dimension. Journal of Physics A: Mathematical and Theoretical, 47(28), p.285001.

(15) Majumdar, S.N., Sabhapandit, S. and Schehr, G., 2015. Dynamical transition in the temporal relaxation of stochastic processes under resetting. Physical Review E, 91(5), p.052131.

(16) Pal, A., 2015. Diffusion in a potential landscape with stochastic resetting. Physical Review E, 91(1), p.012113.

(17) Pal, A., Kundu, A. and Evans, M.R., 2016. Diffusion under time-dependent resetting. Journal of Physics A: Mathematical and Theoretical, 49(22), p.225001.

(18) Pal, A., Chatterjee, R., Reuveni, S. and Kundu, A., 2019. Local time of diffusion with stochastic resetting. Journal of Physics A: Mathematical and Theoretical, 52(26), p.264002.

(19) Eule, S. and Metzger, J.J., 2016. Non-equilibrium steady states of stochastic processes with intermittent resetting. New Journal of Physics, 18(3), p.033006.

(20) Evans, M.R. and Majumdar, S.N., 2018. Effects of refractory period on stochastic resetting. Journal of Physics A: Mathematical and Theoretical. 52 01LT01.

(21) Bodrova, A.S., Chechkin, A.V. and Sokolov, I.M., 2019. Scaled Brownian motion with renewal resetting. Physical Review E, 100(1), p.012120.

(22) Bodrova, A.S., Chechkin, A.V. and Sokolov, I.M., 2019. Nonrenewal resetting of scaled Brownian motion. Physical Review E, 100(1), p.012119.

(23) Basu, U., Kundu, A. and Pal, A., 2019. Symmetric exclusion process under stochastic resetting. Physical Review E, 100(3), p.032136.

(24) Pal, A., Kusmierz, L. and Reuveni, S., 2019. Time-dependent density of diffusion with stochastic resetting is invariant to return speed. Physical Review E, 100(4), p.040101. 
(25) Pal, A., Kusmierz, L. and Reuveni, S., 2019. Invariants of motion with stochastic resetting and space-time coupled returns. New Journal of Physics, 21(11), p.113024.

(26) Bodrova, A.S. and Sokolov, I.M., 2020. Resetting processes with noninstantaneous return. Physical Review E, 101(5), p.052130.

(27) Maso-Puigdellosas, A., Campos, D. and Mendez, V., 2019. Transport properties of random walks under stochastic noninstantaneous resetting. Physical Review E, 100(4), p.042104.

(28) Redner, S., 2007. A Guide to First-Passage Processes. A Guide to First-Passage Processes, by Sidney Redner, Cambridge, UK: Cambridge University Press, 2007.

(29) Bray, A.J., Majumdar, S.N. and Schehr, G., 2013. Persistence and first-passage properties in nonequilibrium systems. Advances in Physics, 62(3), pp.225-361.

(30) Ray, S., Mondal, D. and Reuveni, S., 2019. Peclet number governs transition to acceleratory restart in drift-diffusion. Journal of Physics A: Mathematical and Theoretical, 52(25), p.255002.

(31) Pal, A. and Prasad, V.V., 2019. First passage under stochastic resetting in an interval. Physical Review E, 99(3), p.032123.

(32) Durang, X., S. Lee, L. Lizana, and J.H. Jeon. 2019. First-passage statistics under stochastic resetting in bounded domains. J. Phys. A Math. Theor., 52, p.224001.

(33) Kusmierz, L., Majumdar, S.N., Sabhapandit, S. and Schehr, G., 2014. First order transition for the optimal search time of Lévy flights with resetting. Physical review letters, 113(22), p.220602.

(34) Kusmierz, L., \& Gudowska-Nowak, E. (2015). Optimal first-arrival times in Lévy flights with resetting. Physical Review E, 92(5), 052127.

(35) Reuveni, S., 2016. Optimal stochastic restart renders fluctuations in first passage times universal. Physical review letters, 116(17), p.170601. 
(36) Pal, A. and Reuveni, S., 2017. First Passage under Restart. Physical review letters, 118(3), p.030603.

(37) Chechkin, A. and Sokolov, I.M., 2018. Random search with resetting: a unified renewal approach. Physical review letters, 121(5), p.050601.

(38) Pal, A., Eliazar, I. and Reuveni, S., 2019. First passage under restart with branching. Physical review letters, 122(2), p.020602.

(39) Pal, A. and Prasad, V.V., 2019. Landau-like expansion for phase transitions in stochastic resetting. Physical Review Research, 1(3), p.032001.

(40) Pal, A., Kusmierz, L. and Reuveni, S., 2019. Search with home returns provides advantage under high uncertainty. arXiv preprint arXiv:1906.06987.

(41) Besga, B., Bovon, A., Petrosyan, A., Majumdar, S. and Ciliberto, S., 2020. Optimal mean first-passage time for a Brownian searcher subjected to resetting: experimental and theoretical results. arXiv preprint arXiv:2004.11311.

(42) Dufresne, E.R., Spalding, G.C., Dearing, M.T., Sheets, S.A. and Grier, D.G., 2001. Computer-generated holographic optical tweezer arrays. Review of Scientific Instruments, 72(3), pp.1810-1816.

(43) Polin, M., Ladavac, K., Lee, S.H., Roichman, Y. and Grier, D.G., 2005. Optimized holographic optical traps. Optics Express, 13(15), pp.5831-5845.

(44) Grier, D.G. and Roichman, Y., 2006. Holographic optical trapping. Applied optics, 45(5), pp.880-887.

(45) Crocker, J.C. and Grier, D.G., 1996. Methods of digital video microscopy for colloidal studies. Journal of colloid and interface science, 179(1), pp.298-310.

(46) See Supporting Information for experimental details and theoretical derivations. 
(47) Husain, K. and Krishna, S., 2016. Efficiency of a Stochastic Search with Punctual and Costly Restarts. arXiv preprint arXiv:1609.03754.

(48) Abramowitz, M. and Stegun, I.A. eds., 1948. Handbook of mathematical functions with formulas, graphs, and mathematical tables (Vol. 55). US Government printing office.

(49) Bodrova, A.S. and Sokolov, I.M., 2020. Two-and three dimensional Brownian motion under non-instantaneous resetting. arXiv preprint arXiv:2002.11753.

(50) Redding, N.J., 1999. Estimating the parameters of the K distribution in the intensity domain (No. DSTO-TR-0839).

(51) Long, M.W., 1975. Radar reflectivity of land and sea. Lexington, Mass., DC Heath and Co., 1975. 390 p.

(52) Roichman, Y., Wong, V. and Grier, D., 2007. Colloidal transport through optical tweezer arrays. Phys. Rev. E., 75(1), p011407.

(53) Firnkes, M., Pedone, D., Knezevic, J., Doblinger, M. and Rant, U., 2010. Electrically facilitated translocations of proteins through silicon nitride nanopores: conjoint and competitive action of diffusion, electrophoresis, and electroosmosis. Nano letters, 10(6), pp.2162-2167.

(54) Branton, D., Deamer, D.W., Marziali, A., Bayley, H., Benner, S.A., Butler, T., Di Ventra, M., Garaj, S., Hibbs, A., Huang, X. and Jovanovich, S.B., 2010. The potential and challenges of nanopore sequencing. In Nanoscience and technology: A collection of reviews from Nature Journals (pp. 261-268).

(55) Tu, L.C., Fu, G., Zilman, A. and Musser, S.M., 2013. Large cargo transport by nuclear pores: implications for the spatial organization of FG-nucleoporins. The EMBO journal, 32(24), pp.3220-3230.

(56) Bezrukov, S.M., Kullman, L. and Winterhalter, M., 2000. Probing sugar translocation through maltoporin at the single channel level. FEBS letters, 476(3), pp.224-228. 
(57) Ghale, G., Lanctot, A.G., Kreissl, H.T., Jacob, M.H., Weingart, H., Winterhalter, M. and Nau, W.M., 2014. Chemosensing ensembles for monitoring biomembrane transport in real time. Angewandte Chemie International Edition, 53(10), pp.2762-2765.

(58) Grünwald, D. and Singer, R.H., 2010. In vivo imaging of labelled endogenous $\beta$-actin mRNA during nucleocytoplasmic transport. Nature, 467(7315), p.604.

(59) Ma, J., Liu, Z., Michelotti, N., Pitchiaya, S., Veerapaneni, R., Androsavich, J.R., Walter, N.G. and Yang, W., 2013. High-resolution three-dimensional mapping of mRNA export through the nuclear pore. Nature communications, 4, p.2414.

(60) Iyer-Biswas, S., Wright, C.S., Henry, J.T., Lo, K., Burov, S., Lin, Y., Crooks, G.E., Crosson, S., Dinner, A.R. and Scherer, N.F., 2014. Scaling laws governing stochastic growth and division of single bacterial cells. Proceedings of the National Academy of Sciences, 111(45), pp.15912-15917.

(61) Ingraham, J.L., Maaloe, O. and Neidhardt, F.C., 1983. Growth of the bacterial cell. Sinauer Associates.

(62) Amir, A., 2014. Cell size regulation in bacteria. Physical Review Letters, 112(20), p.208102.

(63) Osella, M., Nugent, E. and Lagomarsino, M.C., 2014. Concerted control of Escherichia coli cell division. Proceedings of the National Academy of Sciences, 111(9), pp.3431-3435.

(64) Cooper, S., 1991. Bacterial growth and division: biochemistry and regulation of the division cycle of prokaryotes and eukaryotes.

(65) Metzler, R., Redner, S. and Oshanin, G., 2014. First-Passage Phenomena and Their Applications (Vol. 35). Singapore: World Scientific.

(66) Fuchs, J., Goldt, S. and Seifert, U., 2016. Stochastic thermodynamics of resetting. EPL (Europhysics Letters), 113(6), p.60009. 
(67) Pal, A. and Rahav, S., 2017. Integral fluctuation theorems for stochastic resetting systems. Physical Review E, 96(6), p.062135.

(68) Gupta, D., Plata, C.A. and Pal, A., 2020. Work fluctuations and Jarzynski equality in stochastic resetting. Physical Review Letters, 124(11), p.110608. 
Graphical TOC Entry

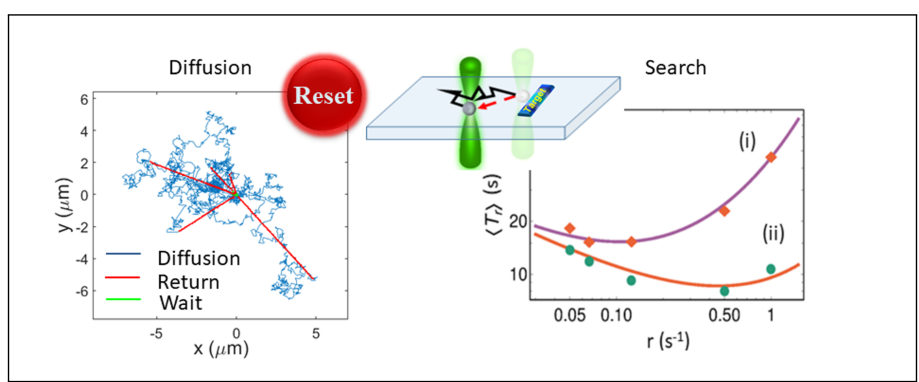

